\title{
An Initial Position Estimation For Switched Reluctance Motor At Standstill
}

\author{
Lei Zhang*, Yue $\mathrm{Yu}^{+}$ \\ * Nanjing Xiao Zhuang University, Nanjing, China \\ *Nanjing College of Information Technology, Nanjing, China
}

Keywords: switched reluctance motor; sensorless; initial position; pulse time

\begin{abstract}
The paper presents a new initial position estimation strategy of switched reluctance motor based on time threshold pulse injection. According to the relationship of the inductance, the phase current and the rotor position, at the stationary state, the high frequency pulse is injected to the phase at the same time, the peak value of the pulse current rise is equal, the rotor initial partition is estimated by compare with the rise time of the pulse current. The proposed method does not require additional hardware or memory. At last, the simulation model is built, and the results verify the correctness and feasibility of the proposed algorithm.
\end{abstract}

\section{Introduction}

Switched reluctance motor (switch reluctance motor SRM) is a new mechanical and electrical integration of adjustable speed motor, the doubly salient structure, concentrated winding is wound on the stator, No permanent magnet material and winding on the rotor, and has the advantages of simple structure, stout, starting torque, wide speed range, high operating efficiency ,advantages[1]. The traditional photoelectric, electromagnetic and magnetic sensing type position sensor increases the cost of the system and reduce the reliability of the systems [2], limiting the application of SRM. Therefore, no position sensor technology research become the whole field is a potential direction.

SRM sensorless control method has many, in recent years, the experts of domestic and foreign have carried out extensive research on the position sensorless technique [3]. Take advantage of the signals collected by sensors, and calculate the inductance and flux linkage, and estimate the rotor position . The initial position estimation of SRM is studied[411]. The high frequency pulse is injected for each phase winding at the same time [4-7], the initial position is estimate $d$ by comparison of the peak value of the pulse current, the algorithm is simple, but in the maximum and minimum area, the inductance change slightly, the peak value of pulse current variation is small, the position estimation error magnify. Based on literature [4-7], improve the accuracy of position estimation by robust analysis[8].The initial position estimation method is proposed by compare pulse current peak value with the current threshold in [9], the method is simple and easy to implement. The initial position of the SRM is estimated by the relationship between the bootstrap circuit capacitor charging current amplitude and the phase inductance [10-11]. The full cycle inductance method is proposed to realize the initial position estimation, and the starting control of the no position sensor, the fault tolerance is also studied [12].

From the domestic and foreign literature, the initial position estimates of SRM is based on pulse injection method, by detecting the peak pulse current, estimate the initial position of the switched reluctance motor from different angles, but in the region of little difference inductance, the current pulse response peak is relatively close, so comparison of pulse current peak value for the initial position estimates of SRM, position estimates error becomes large. Therefore, improve the pulse injected for the initial position estimation in the paper, proposes a method based on pulse injection time threshold, the initial position is estimated by comparing with the time of pulse current rising, relationship between the time of pulse current rising and rotor position. Solve the position of position estimation error in subtle inductance, at the same time, but also improve the reliability of motor starting. Finally, the simulation and experiment verify the feasibility of the proposed method.

\section{Principle of SRM sensorless}

\subsection{Basic principle of pulse injection method}

The control system of the switched reluctance motor, the common power converter is the asymmetrical half bridge structure, and the pulse injected to each phase is convenient. When the motor phase winding is imposed phase voltage, ignore the saturation and mutual inductance, and hysteresis loss and eddy current loss of motor, the motor voltage balance equation for:

$$
u_{k}=R_{k} i_{k}+L_{k}(\theta) \frac{d i_{k}}{d t}+i_{k} \omega \frac{d L_{k}(\theta)}{d \theta}
$$

Where $u_{k}$ is the end voltage of the phase winding, $R_{k}$ is the phase resistance, $i_{k}$ is the phase current,$L_{k}$ is the phase inductance, $\omega$ is speed, $\theta$ is the rotor position angle, $k=1,2 \ldots ., m, m$ is the phase number.

When the motor is stalling, the frequency of the injected pulse signal is high, the current rise time is short, the current peak is small, and the voltage drop of the motor winding is neglected, equation (1) can be simplified as: 


$$
\begin{aligned}
& u_{k} \approx L_{k}(\theta) \frac{d i_{k}}{d t} \\
& L_{k}(\theta) \approx U_{k} \frac{\Delta T}{\Delta I}
\end{aligned}
$$

According to equation (3), when the bus voltage and the injected high frequency pulse frequency duty ratio is fixed, the motor phase inductance and pulse response current variation is inversely proportional, and the motor phase inductance contains the motor position information, so the initial position is estimated by comparing the magnitude of the pulse current based on current threshold .Because the pulse current is small, and the existence of inertia and friction, the motor keep in the stationary state .In pulse injection process, the phase voltage is positive, the pulse current rise linear, the phase voltage is negative, the pulse current fall linear. Because the phase inductance do not change, the current rising slope and down slope is equal.

\subsection{Basic principle of time threshold}

According to equation(2), there is a quantitative mathematical relationship between the phase inductance, the impulse response current, and the current rise time. The formula (2) can be deformed:

$$
\Delta T \approx L_{k}(\theta) \frac{\Delta I}{U_{k}}
$$

Can be seen from the formula (4), when the bus voltage $U_{k}$ of the motor and the current change $\Delta I$ are constant value, the phase current rise time $\Delta T$ is proportional to the phase inductance $L_{k}$. According to the previous analysis, the phase inductance contains the rotor position information, so it can detect the position of the motor rotor indirectly by the method of detecting the rise time of the impulse response current.

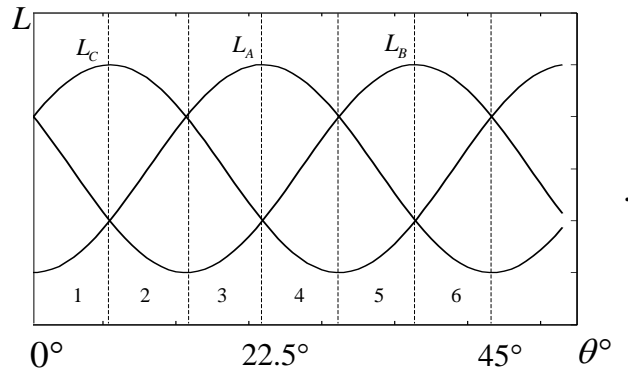

Fig.1 Inductance curve division

Fig. 1 shows that the inductance curve and rotor position are monotone function in each partition. The logical relationship between the rise time of the three phase current and the initial starting phase is provided in Table 1. For example in the 3 partition, at the same time, the difference duty cycle high frequency pulse is injected to the three-phase, when the pulse current reach the maximum variation of pulse current, and detect pulse current rise time, and compare the magnitude of the three-phase current rise time. The result of $\Delta T_{B}<\Delta T_{C} \leq \Delta T_{A}$ is obtained, the motor rotor locate currently in 3 partition, so the initial conduction phase is the B phase.

Table 1 . The logic relation of the three measure

\begin{tabular}{|c|c|c|}
\hline Initial phase & Current rise time & Position interval \\
\hline $\mathrm{A}$ & $\Delta T_{A}<\Delta T_{B} \leq \Delta T_{C}$ & $0^{\circ} \leq \theta_{A}<7.5^{\circ}$ \\
\hline $\mathrm{A}$ & $\Delta T_{B} \leq \Delta T_{A}<\Delta T_{C}$ & $7.5^{\circ} \leq \theta_{A}<15^{\circ}$ \\
\hline $\mathrm{B}$ & $\Delta T_{B}<\Delta T_{C} \leq \Delta T_{A}$ & $15^{\circ} \leq \theta_{A}<22.5^{\circ}$ \\
\hline $\mathrm{B}$ & $\Delta T_{C} \leq \Delta T_{B}<\Delta T_{A}$ & $22.5^{\circ} \leq \theta_{A}<30^{\circ}$ \\
\hline $\mathrm{C}$ & $\Delta T_{C}<\Delta T_{A} \leq \Delta T_{B}$ & $30^{\circ} \leq \theta_{A}<37.5^{\circ}$ \\
\hline $\mathrm{C}$ & $\Delta T_{A} \leq \Delta T_{C}<\Delta T_{B}$ & $37.5^{\circ} \leq \theta_{A}<45^{\circ}$ \\
\hline
\end{tabular}

\subsection{Comparison and Analysis}

In the actual process, the winding pressure drop and the saturation effect can not be ignored, the current cannot rise linearly in stationary state. Fig. 2 shows the pulse current response waveform of the motor, the solid line shows the pulse current response waveform for the pulse injected method based on current threshold by comparing the magnitude of the three-phase pulse current, the dotted line shows the pulse current response waveform of motor for the pulse injection method of time threshold by comparing the magnitude of the three-phase pulse current rise time. According to Fig.2, the value of the two phases inductance is close near the intersection region of the two phase inductance curves ,to the pulse injected method based on the current threshold, because of the fixed duty cycle and high frequency pulse, the peak value of the pulse current is scarcely less, and the low degree of distinction, and amplify the initial position estimation error .To the pulse current injected method based on the time threshold, and fixed pulse current amplitude, and the slope of the pulse current curve is reduced, and amplify the pulse current rise time, and increase the degree of detection, improve the accuracy of initial position estimation.

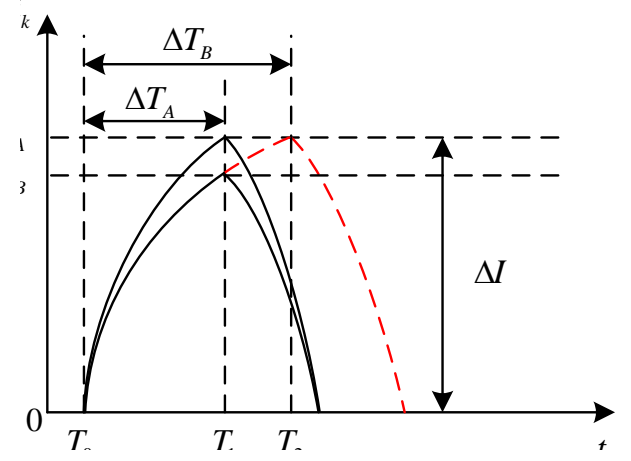

Fig.2 Schematic diagram of pulse current response Sampling errors may be produced in the process of pulse current sampling, Fig.3 shows the diagrammatic sketch of the peak value of the pulse current is obtained at a certain position in, according to equation (3), the inductance value calculated is small, the initial position estimation error will be amplified, the most serious consequence cause the failure of the initial 
partition, which ultimately leads to the failure of the motor starting, and even reverse.

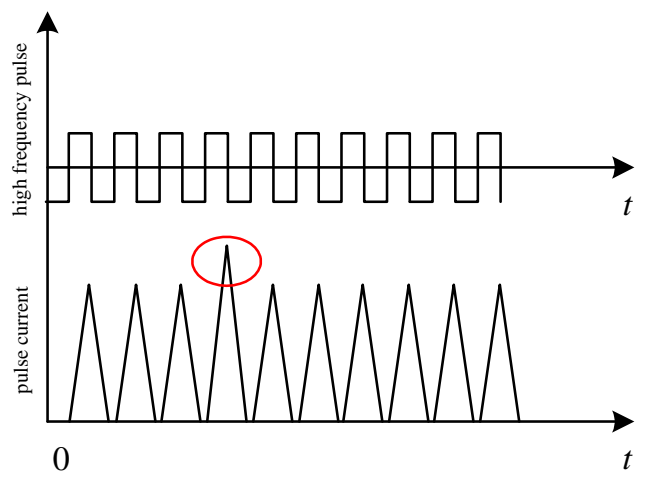

Fig.3 Pulse current error

It can avoid the occurrence of this phenomenon by using the pulse injection method based on the time threshold. The amplitude of pulse current is detected real-time, when the pulse current reaches the fixed pulse current amplitude, turn off the switch tube immediately, and the pulse injection phase of the motor is in the phase of the continuous flow.

\subsection{The fixed pulse current amplitude}

In a static state, if the fixed pulse current amplitude is larger, the motor revolve by injected pulse current, and destroy the initial state of the motor, and even cause the motor to reverse. The minimum value of the pulse current is determined by the current sensor, it is necessary to make sure that the current is in the range of detection of the current sensor. Therefore, it is very important to select the fixed pulse current amplitude

Ignore the saturation of the motor, the response current of the injected pulse is small, It is considered that the magnetic circuit of the motor is linear, the electromagnetic torque of a phase can be given as:

$$
T_{e} \approx \frac{1}{2} i^{2} \frac{d L}{d \theta}
$$

Where $\frac{d L}{d \theta}$ is the phase inductance variation slope, the magnetic path of SRM is linear similarly, the phase inductance of the motor can be calculated by linear model, the gradient of the phase inductance can be expressed as :

$$
\frac{d L}{d \theta}=\frac{L_{\max }-L_{\min }}{\beta_{s}}
$$

Where $L_{\max }$ is the maximal value of phase inductance, $L_{\min }$ is the minimal value of phase inductance, $\beta_{s}$ is the polar arc of motor stator, $T_{L}$ is the load torque of SRM, $i_{\max }$ is the maximum current value of motor.

$$
\Delta I=i_{\max }
$$

and

$$
T_{e}<T_{L}
$$

From (5) and (6) and (7) and (8), the prescript value of the pulse current can be expressed as :

$$
\Delta I<\sqrt{\frac{2 T_{L} \beta_{s}}{L_{\max }-L_{\min }}}
$$

\subsection{Influence of sampling frequency}

In the course of the experiment, the sampled data is a series of continuous scattered points, The pulse current peak is often not the current threshold, so it is necessary to analyze the error caused by the sampling frequency.

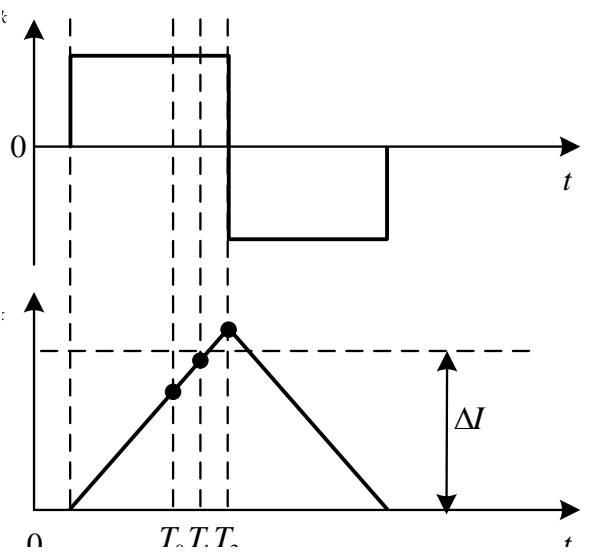

Fig.4 Sketch of error analysis

In Fig.4, at the T1 moment, the current value of the sample has not yet reached the preset current peak value, and the motor phase winding keep on the positive voltage, before the arrival of the next sampling period, the peak value of the phase current of the motor has reached the preset fixed pulse current amplitude, at the T2 moment, the current peak value of the sample is higher than the preset fixed pulse current amplitude , the switch tube turn off, the phase winding flow residual current. From the above analysis, error is not greater than one sampling period by time threshold pulse injection method

$$
T_{e r r}<\frac{1}{f}
$$

Where $t$ is the current sampling frequency.

\section{Simulation analysis}

In order to verify the validity of the theory, the control strategy simulation is proposed in the paper by Matlb/Simulink.

The three-phase 12/8 SRM, the power rating1.5kw, the rated speed $1500 \mathrm{r} / \mathrm{min}$, in the process of simulation, the bus voltage $U_{d c}=30 \mathrm{~V}$, the maximum rise current $\Delta I=0.5 \mathrm{~A}$.

Three phase pulse current waveform are shown at the different initial positions in Fig.5. According to the Fig,5, the time of the pulse current reaching to the preset fixed pulse current amplitude is not the same at the different initial positions for the three-phase, so just collect the rise time of each phase current and compare their size, decide the current location of the rotor.Fig.5 (b) as an example, we can seen $T_{C}<T_{B}<T_{A}$ 
from the Fig.5 (b), lookup from table 1, it can be seen that the current rotor is in partition 3, in accordance with the preset a phase angle of 18 degrees, the same as the above theoretical analysis results.
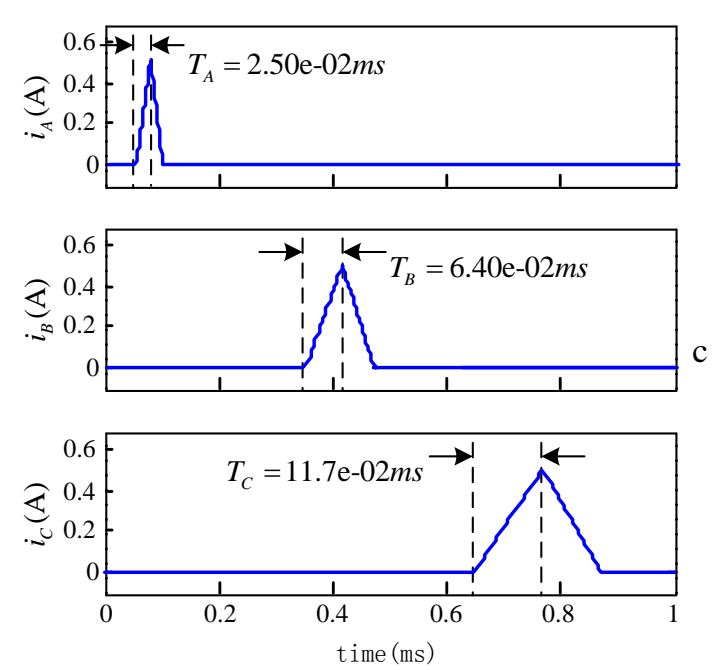

(a) The waveform of pulse current rising at the initial angle of 4 degrees
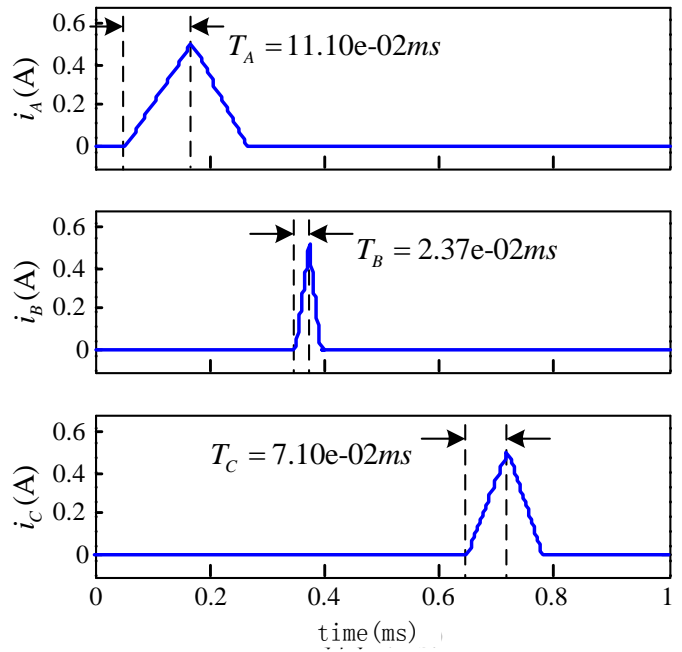

(b) The waveform of pulse current rising at the initial angle of 18 degrees
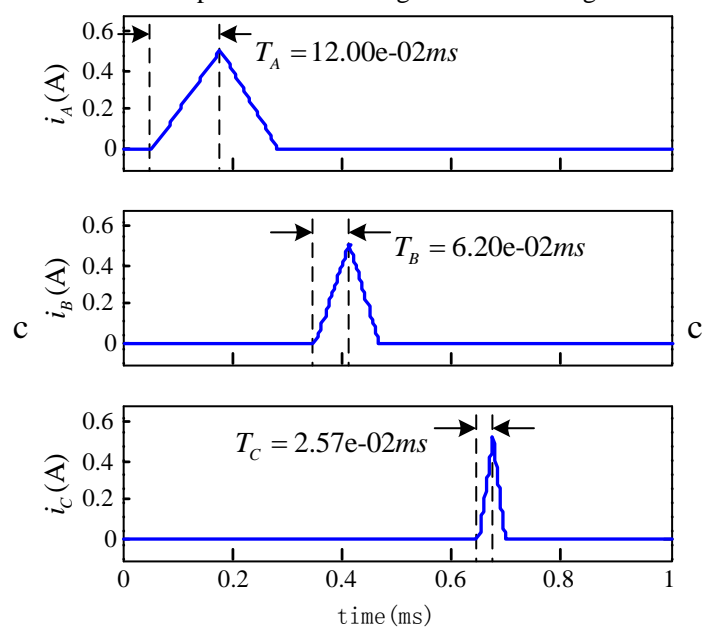

(c) The pulse current rising waveform at the initial angle of 22.5 degrees
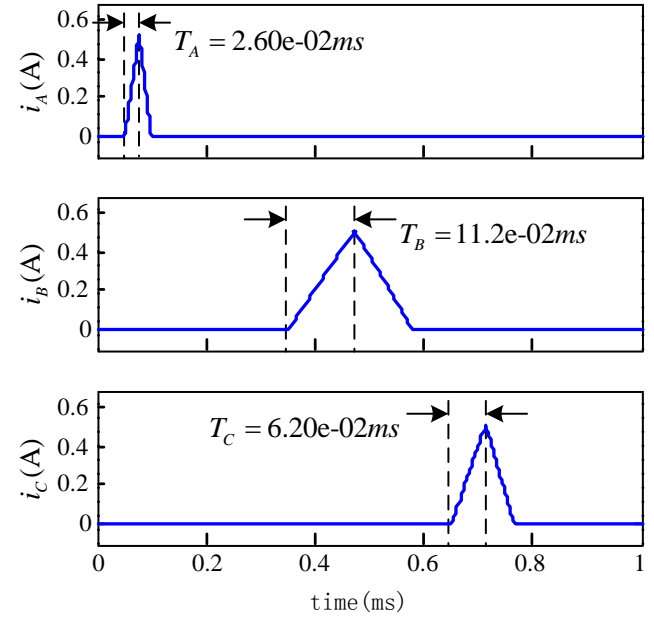

(d) The pulse current rising waveform at the initial angle of 40.5 degrees

Fig.5 Three phase pulse current rise time at different initial positions

In order to verify that the initial position of the rotor can be determined by detecting the rise time of the three-phase current, in accordance with the above simulation method, the rise time of three phase currents is simulated an interval of 2 degrees in table 2.

Table 2. The three phase current rise time

\begin{tabular}{cccccc}
\hline$\theta_{A}\left(^{*}\right.$ & $T_{A}(m s)$ & $\theta_{s}\left({ }^{*}\right)$ & $T_{s}(m s)$ & $\theta_{c}\left({ }^{*}\right)$ & $T_{c}(m s)$ \\
\hline 0.5 & $2.19 \mathrm{e}-02$ & 30.5 & $8.90 \mathrm{e}-02$ & 15.5 & $9.50 \mathrm{e}-02$ \\
2.5 & $2.29 \mathrm{e}-02$ & 32.5 & $7.40 \mathrm{e}-02$ & 17.5 & $10.70 \mathrm{e}-02$ \\
4.5 & $2.60 \mathrm{e}-02$ & 34.5 & $6.20 \mathrm{e}-02$ & 19.5 & $12.00 \mathrm{e}-02$ \\
6.5 & $3.30 \mathrm{e}-02$ & 36.5 & $4.60 \mathrm{e}-02$ & 21.5 & $12.80 \mathrm{e}-02$ \\
8.5 & $4.60 \mathrm{e}-02$ & 38.5 & $3.30 \mathrm{e}-02$ & 23.5 & $12.80 \mathrm{e}-02$ \\
10.5 & $6.20 \mathrm{e}-02$ & 40.5 & $2.57 \mathrm{e}-02$ & 25.5 & $12.00 \mathrm{e}-02$ \\
12.5 & $7.40 \mathrm{e}-02$ & 42.5 & $2.37 \mathrm{e}-02$ & 27.5 & $10.70 \mathrm{e}-02$ \\
14.5 & $8.90 \mathrm{e}-02$ & 44.5 & $2.26 \mathrm{e}-02$ & 29.5 & $9.50 \mathrm{e}-02$ \\
16.5 & $10.10 \mathrm{e}-02$ & 1.5 & $2.26 \mathrm{e}-02$ & 31.5 & $8.20 \mathrm{e}-02$ \\
18.5 & $11.40 \mathrm{e}-02$ & 3.5 & $2.47 \mathrm{e}-02$ & 33.5 & $6.70 \mathrm{e}-02$ \\
20.5 & $12.50 \mathrm{e}-02$ & 5.5 & $2.89 \mathrm{e}-02$ & 35.5 & $5.40 \mathrm{e}-02$ \\
22.5 & $13.0 \mathrm{e}-02$ & 7.5 & $3.90 \mathrm{e}-02$ & 37.5 & $3.90 \mathrm{e}-02$ \\
\hline
\end{tabular}

The inductive period is 45 degrees, and 22.5 degree symmetrical relation, so the table 2 shows the simulation results of half cycle. The relationship between the rise time of the pulse current and the rotor position is shown in Fig.6, the curves of the pulse current rise time and the inductance curve are consistent by comparison with Fig. 1, so as to verify the correctness of the above theory. 


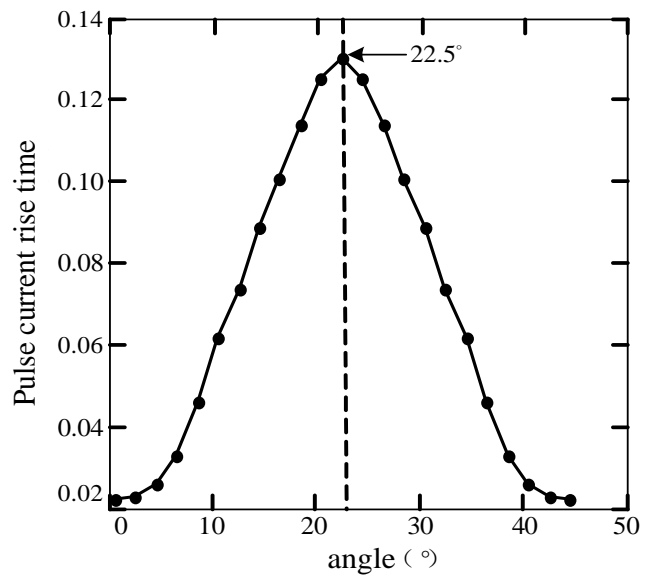

Fig.6 Relationship between the rise time of pulse current and rotor position

\section{Conclusion}

In this paper, a initial position estimation method based on the pulse injected time threshold is proposed for SRM, the initial position detecting system is established. the preset fixed pulse current amplitude, and detect the time of the each pulse current reaching to the preset fixed pulse current amplitude ,by comparing the time to estimate the initial partition of the rotor .The position estimation accuracy is great than that of the current threshold pulse injection method, the experiment platform is established based on dSPACE1104. The feasibility and validity proposed method is verified by both simulation and experiment. The result shows a nice dynamic performance of the method.The proposed initial position estimation method is simple and suitable for without inversion. not complex operations and additional hardware circuitry.

\section{Acknowledgment}

The author would like to thank the support of the Natural Science Foundation of JiangSu. Grant No: BK20151574.

\section{References}

[1] Wu Hong xing, JiHeng, NiTian, et al. Summary of Novel Switched Reluctance Motor Development[J]. Micro Motors,2011,44(1): 78-83

[2] Tom Per1, Iqbal Husain, Malik Elbuluk. Design Trends and Trade - offs for Sensorless Operation of Switched Reluctance Motor Drives[C]. IEEE Industry Application Conference, 13th IAS Annual Meeting, $1995: 278$ 285

[3] Ehsani M, Fahimi B. Elimination of position sensors in switched reluctance motor drives : state of the art and future trends[J]. IEEE Trans. on Industry Electronics, 2002, 49(1) : 40-48.

[4] H.Gao,F.RSalmasi,and M.Ehsani. Senorless control of SRM at standstill[C].In Proc. APEC'00,2000:850-856.

[5] Gao Hongwei. Sensorless control of the switched reluctance motor drive at standstill and near-zero
speed[D]:Texas A\&M University,2001.

[6] Krishnamurthy M.Edrington C S, Fahimi B. Prediction of rotor position at standstill and shaft condition in switched reluctance machines[J].IEEE Transaction on Power Electronics, 2006,21(1):225-233.

[7] Amiri H, Afjei E,Ataei S. A novel sensorless technique for a new field assisted switched reluctance motor[C].International Symposium on Power Electronics ,Electrical Drivers, Automation and Motion,2008:1077-1081.

[8] Liu Weiguo, Song Shoujun, Wue Sweafer. Initial Position Estimation of Sensorless Switched Reluctance Motor[J]. Proceedings of the CESS, 2009, 29(24): 91-97.

[9] Krishnamurthy M,Edrington C.C,Fahimi B. Prediction of rotor position at standstill and rotating shaft conditions in switched reluctance machines .IEEE Trans.Power Electron,2006,21(1):225-233.

[10] L.Shen,J.H.Wu, and S.Y.Yang. Initial position estimation in SRM using bootstrap circuit without predefined inductance parameters[J].IEEE Trans .Power Electron ,2011,26(9):2449-2456.

[11] Shen Lei,Wu Jianhua,Yang Shiyou. A Novel Estimation Method for Initial Position of Switched Reluctance Motor Based on Bootstrap Circuit[J]. Proceeding of the CESS, 2011,31(6):98-102.

[12] Cai Jun, Deng Zhiquan. Sensorless Control of Switched Reluctance Motor Based on Full-cycle Inductance Method[J]. Transation of China Electrotechnical Society, 2013, 28(2): 145-153. 